\title{
Imaging Irreversible Transformations with Movie-Mode Dynamic Transmission Electron Microscopy
}

\author{
Joseph T. McKeown, Melissa K. Santala, Tian Li, John D. Roehling, and Geoffrey H. \\ Campbell
}

Materials Science Division, Lawrence Livermore National Laboratory, Livermore, CA, USA

In situ transmission electron microscopy (TEM) has been utilized for decades to image materials processes at high spatial resolution. Yet the relevant dynamics of many of these processes often remain elusive, as they unfold too rapidly to discern at small spatial scales using conventional TEM imaging conditions. For example, consider a transformation front moving with a relatively low velocity of $0.1 \mathrm{~mm} / \mathrm{s}$. In situ TEM imaging conducted with conventional acquisition rates of 30 frames/s corresponds to a temporal resolution of $\sim 33 \mathrm{~ms}$ and, limited by motion blur, provides a spatial resolution of only $\sim 3 \mu \mathrm{m}$. Given the rapid microstructural evolution of many types of irreversible transformation fronts-on the order of $\mathrm{mm} / \mathrm{s}$ to $\mathrm{m} / \mathrm{s}$ - nanosecond temporal resolutions are required to capture these processes.

The dynamic transmission electron microscope (DTEM) at LLNL was developed to enable imaging of transient states during irreversible transformations with nanometer spatial and nanosecond temporal resolutions using a single-shot acquisition mode [1]. Recently, movie-mode DTEM was designed and implemented to provide multiple (up to 9) single-shot acquisitions in under $\sim 1 \mu \mathrm{s}$, yielding frame rates that are on the order of $10^{6}$ times higher than conventional in situ TEM frame rates [2]. Movie-mode DTEM employs an arbitrary waveform generator to deliver a user-defined (pulse duration and spacing) series of electron pulses, allowing complex irreversible transformation events to be tracked across microsecond timescales. Movie-mode DTEM acquisitions result in higher data throughput with reduced uncertainty. Here, we will provide an overview of movie-mode DTEM instrumentation and operation, with examples of its application to various materials science problems, including rapid alloy solidification (Figure 1), where solid-liquid interface evolution is monitored, and amorphous-crystalline phase transformations (Figure 2) involving semiconductor and phase-change materials.

\section{References}

[1] G.H. Campbell, J.T. McKeown, and M.K. Santala, Appl. Phys. Rev. 1 (2014) 041101.

[2] T. LaGrange, B.W. Reed, and D.J. Masiel, MRS Bull. 40 (2015) 22.

[3] This work was performed under the auspices of the U.S. Department of Energy, Office of Basic Energy Sciences, Division of Materials Sciences and Engineering for 
FWP SCW0974 by Lawrence Livermore National Laboratory under Contract DEAC52-07NA27344.

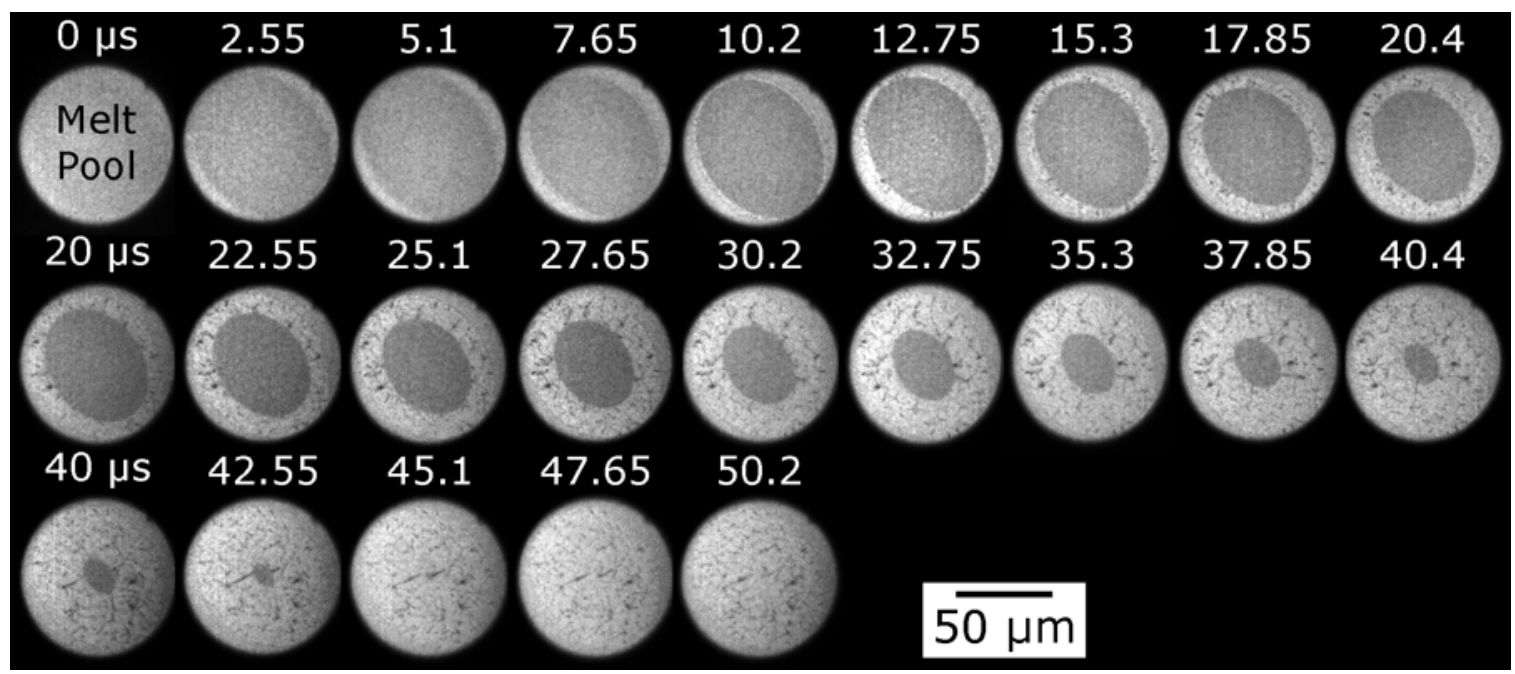

Figure 1: Movie-mode DTEM image series acquired during the rapid solidification of an Al-3at.\%Si alloy following laser melting. The entire solidification process is complete in $\sim 50 \mu \mathrm{s}$. Each row of 9 images is a separate experiment that begins at the delay time above the leftmost image. Prior to movie mode, this entire data set would have required 23 separate single-shot experiments as opposed to the 3 movie-mode experiments shown here. Each frame was acquired with a 50-ns electron pulse; the inter-frame spacing was $2.5 \mu \mathrm{s}$.

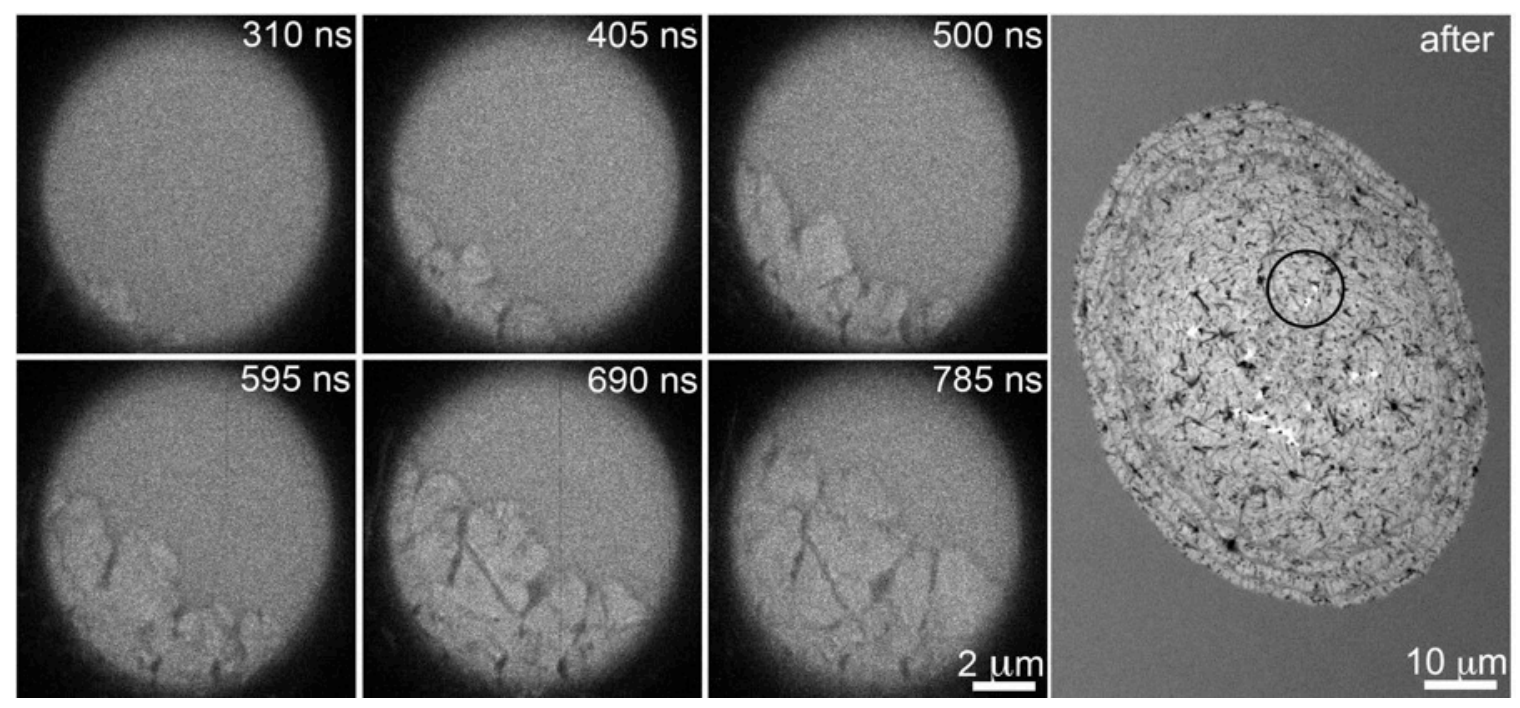

Figure 2: Movie-mode DTEM image series of laser crystallization of amorphous Ge. The 95-ns inter-frame time allows the crystallization front, moving at $10 \mathrm{~m} / \mathrm{s}$, to be imaged. The region imaged in the 20-ns exposures (left) is circled in the conventional TEM image (right). 